\title{
Energia digestível para alevinos de tilápia-do-nilo (Oreochromis niloticus)
}

\author{
Wilson Rogério Boscolo , Aldi Feiden ${ }^{1}$, Altevir Signor ${ }^{2}$, Arcangelo Augusto Signor ${ }^{2}$, Jandir \\ José Bard ${ }^{2}$, Fabio Akira Ishida ${ }^{2}$
}

${ }^{1}$ Curso de Engenharia de Pesca - Unioeste - Campus ToledolParaná.

2 Acadêmico do curso de Engenharia de Pesca da Unioeste - Campus ToledolParaná.

RESUMO - Avaliou-se o efeito de diferentes níveis de energia digestível na dieta sobre o desempenho de alevinos de tilápiado-nilo (Oreochromis niloticus). Utilizaram-se 125 alevinos com peso e comprimento iniciais de 0,62 $\pm 0,12 \mathrm{~g} \mathrm{e} 3,25 \pm 0,25 \mathrm{~cm}$, respectivamente, distribuídos em 25 aquários com capacidade de $30 \mathrm{~L}$, em um delineamento experimental inteiramente casualizado, com cinco tratamentos e cinco repetições, em que a unidade experimental consistiu de um aquário contendo cinco alevinos. As rações, isoprotéicas (30\% de proteína digestível), isofosfóricas e isocalcíticas, foram formuladas para conter 2.900 ; $3.025 ; 3.150 ; 3.275$ e $3.400 \mathrm{kcal} / \mathrm{kg}$ de energia digestível. A quantidade de ração fornecida (quatro vezes ao dia) correspondeu a $10 \%$ da biomassa. Os parâmetros físico-químicos da água (oxigênio dissolvido - OD, pH e condutividade elétrica - CE) foram mensurados semanalmente, à tarde, e a temperatura, diariamente, antes da primeira e da última sifonagem, apresentando médias de $8,00 \pm 0,05 \mathrm{mg} / \mathrm{L} ; 7,91 \pm 0,19 ; 92,11 \pm 2,27 \mu \mathrm{S} / \mathrm{cm}$ e $25,61 \pm 0,90^{\circ} \mathrm{C}$, respectivamente. Ao final do experimento, foram analisadas as médias de peso final, ganho de peso, comprimento final, conversão alimentar aparente, sobrevivência e fator de condição. Não foram observadas diferenças no desempenho de alevinos entre os diferentes tratamentos. Recomenda-se a utilização de 2.900 a $3.400 \mathrm{kcal}$ de $\mathrm{ED} / \mathrm{kg}$ na ração de alevinos de tilápia-do-nilo.

Palavras-chave: desempenho, energia, Oreochromis niloticus, tilápia

\section{Digestible energy for Nile tilapia (Oreochromis niloticus) fingerlings}

\begin{abstract}
The different levels of digestible energy on the performance of Nile tilapia (Oreochromis niloticus) fingerlings were evaluated. One hundred and twenty-five fingerlings averaging initial length and weight of $0.62 \pm 0.12$ and $3.25 \pm 0.25 \mathrm{~cm}$ were allotted to $2530 \mathrm{~L}$-aquariums, as a completely randomized design with five treatments and five replicates (an aquarium with five fingerlings was considered the experimental unit). The diets, formulated to be isonitrogenous (30\% of digestible protein), isophosphorous and isocalcium, contained 3,300, 3,525, 3,750, 3,975, and $4,200 \mathrm{kcal} / \mathrm{kg}$ of digestible energy. The amount of diet was fed of $10 \%$ of biomass four times a day. The abiotic parameters $(\mathrm{pH}$, dissolved oxigen - DO, and electric condutivity - EC) were weekly measured at afternoon and the temperature was was daily mensured. The following values were observed: $\mathrm{pH}$ of $7.91 \pm 0.19$, DO of $8.00 \pm 0.05 \mathrm{mg} / \mathrm{L}, \mathrm{EC}$ of $92.11 \pm 2.27 \mu \mathrm{S} / \mathrm{cm}$, and temperature of $25.61 \pm 0.90^{\circ} \mathrm{C}$. At the end of experiment, means of final weight, weight gain, final length, apparent feeding conversion, survival rate and condition factor were analyzed. No significant differences on fingerling perfomance were found across the treatments. It can be included from 2,900 to $3,400 \mathrm{kcal}$ of $\mathrm{ED} / \mathrm{kg}$ in the diet of Nile tilapia fingerlings.
\end{abstract}

Key Words: energy, Oreochromis niloticus, tilapia, performance

\section{Introdução}

As tilápias destacam-se na aqüicultura mundial e, atualmente, formam o segundo maior grupo de peixes cultivados, por apresentarem crescimento rápido e rusticidade (Hayashi et al., 1999a), fácil reprodução, manipulação de sexo, carne de ampla aceitação no mercado consumidor, pela inexistência de espinhos em forma de "y" no seu filé e fácil industrialização (Hildsorf, 1995). A tilápia, de hábito alimentar onívoro, apresenta eficiente utilização dos carboidratos e, em decorrência do aumento de sua produ- ção, muitos aspectos de sua nutrição têm sido estudados (Barros et al., 2002; Pezzato et al., 2002).

A relação energia:proteína e a disponibilidade de nutrientes devem ser adequadas às exigências da espécie para que os peixes apresentem boas taxas de crescimento (Meurer et al., 2002; Hayashi et al., 2002; Boscolo et al., 2004). A elevada disponibilidade de energia nas rações resulta em baixa ingestão de proteína e, conseqüentemente, de nutrientes essenciais (Chou \& Shiau, 1996; Hayashi et al., 2002; Boscolo et al., 2005), ocasionando deposição de gorduras visceral e corporal em várias espécies (MacGoogan \& 
Reigh, 1996; Mukhopadhyay \& Ray, 1997), perda de qualidade da carne, elevada oxidação de ácidos graxos e menor vida de prateleira (Mente et al., 2003).

A deficiência energética nas dietas, no entanto, provoca a sintetização de energia a partir de proteínas - elevando os índices de conversão alimentar e o custo de produção, visto que a proteína é o nutriente mais oneroso (Lowell, 1989)-e promove o aumento de excreção de amônia no ambiente aquático, tornando-se um potencial poluidor (Pezzato et al., 2002).

Os óleos e as gorduras são fontes energéticas facilmente encontradas no mercado e apresentam altas taxas de energia e considerável quantidade de ácidos graxos essenciais (Steffens, 1987; Meurer et al., 2002), de modo que o perfil corporal de ácidos graxos de muitas espécies reflete o da dieta (Lee et al., 2003; Bendiksen et al., 2003; Boscolo et al., 2005). Segundo Sanches (2004), além dos níveis adequados de lipídeos, é fundamental o perfil de ácidos graxos essenciais na dieta para a promoção de bom desempenho dos peixes.

O fornecimento de lipídeos como fonte de energia em dietas para peixes melhora a conversão alimentar (Martino etal., 2002), possui custo acessível e alto nível energético (Jauncey, 2000) e pode influenciar o consumo de alimento (Lee et al., 2002; Boscolo et al., 2005) e melhorar a digestibilidade de alimentos vegetais (Belal \& Assem, 1995). Alguns autores (El-Dahhar \& El-Shazly, 1993; Chou\& Shiau, 1996; Meureretal., 2002)afirmam que a tilápia não utiliza eficientemente os lipídios como fonte energética em níveis acima de 5\% da dieta, aumentando a deposição de gordura corporal.

De acordo com Boscolo etal.(2002), oóleo de soja apresenta $8.485 \mathrm{kcal} / \mathrm{kg}$ de energia digestível e $89,85 \%$ de digestibilidade aparente (CDA), demonstrando que a tilápia absorve eficientemente a energia desta fonte alimentar, como observado por Hanley (1987), ao avaliaremo CDA da EB deóleo animal. Como o alimento é o componente mais oneroso da produção (Lutz, 2000; Boscolo et al., 2001), é importante avaliar as exigências energética e nutricional da tilápia-do-nilo (Oreochromis niloticus) na fase de alevino, visando à obtençao de animais com qualidade para as fases subseqüentes de produção.

Este trabalho foi conduzido com o objetivo de avaliar os efeitos de diferentes níveis de energia digestível na dieta de alevinos de tilápia-do-nilo (O. niloticus) sobre o seu desempenho.

\section{Material e Métodos}

O trabalho foi realizado no Laboratório de Aqüicultura da Universidade Estadual do Oeste do Paraná - Campus de Toledo no período de 20 de março a 16 de abril de 2004

Foram utilizados 125 alevinos de tilápia-do-nilo (O. niloticus) com $0,62 \pm 0,12 \mathrm{~g}$ (peso obtido em balança analítica) e comprimento inicial de $3,25 \pm 0,25 \mathrm{~cm}$ (medido em ictiômetro), distribuídos em 25 aquários com volume útil de $30 \mathrm{~L}(30 \times 30 \times 35 \mathrm{~cm})$, resultando em densidade de 0,17 peixes/L. Utilizou-se delineamento inteiramente casualisado com cinco tratamentos e cinco repetições, considerando a unidade experimental um aquário com cinco alevinos.

Os aquários possuíam sistema de aeração individual, ligados a um soprador de ar central. Diariamente, às 8 e $17 \mathrm{~h}$, realizou-se a sifonagem dos aquários, antes da primeira e da última alimentação, para retirada de sobras de ração e de fezes, substituindo aproximadamente $40 \%$ do volume total duas vezes ao dia.

Os alimentos foram processados em um moinho tipo faca com peneira de $0,5 \mathrm{~mm}$ e as rações experimentais (Tabela 1), isocalcíticas, isofosfóricas e isoprotéicas com $30 \%$ de proteína digestível (Tabela 2 ), foram formuladas para conter níveis de $2.900,3.025,3.150,3.275$ e $3.400 \mathrm{kcal} / \mathrm{kg}$ de energia digestível. O arraçoamento, a 10\% da biomassa do aquário, foi dividido em quatro alimentações diárias $(8,11,14$ e 17 h). Para correção da taxa de arraçoamento, foram realizadas biometrias a cada 10 dias.

Os parâmetros físico-químicos ( $\mathrm{pH}$, condutividade elétrica $[\mu \mathrm{S} / \mathrm{cm}]$ e oxigênio dissolvido $[\mathrm{mg} / \mathrm{L}])$ foram mensurados semanalmente, enquanto a temperatura $\left({ }^{\circ} \mathrm{C}\right)$ foi coletada diariamente pela manhã $(8 \mathrm{~h})$ e à tarde $(17 \mathrm{~h})$, antes da primeira e da última sifonagem, utilizando-se medidores portáteis.

Ao final do período experimental, os peixes foram mantidos em jejum por 12 horas e, após este período, foram efetuadas as medidas individuais de peso (g) e comprimento total $(\mathrm{cm})$ de cada unidade experimental. Foram avaliados o ganho de peso (GP), o comprimento final (CF), a sobrevivência (SO), o fator de condição (FC), o peso final médio (PF) e a conversão alimentar aparente (CA). Os dados foram submetidos à análise de variância a 5\% de significância pelo programa estatístico SAEG (Sistema de Análises Estatísticas e Genéticas) (UFV, 1997).

\section{Resultados e Discussão}

Os parâmetros físico-químicos da água dos aquários experimentais - temperatura $\left(25,61 \pm 0,90^{\circ} \mathrm{C}\right)$, oxigênio dissolvido $(8,00 \pm 0,05 \mathrm{mg} / \mathrm{L}), \mathrm{pH}(7,91 \pm 0,19)$ e condutividade elétrica $(92,11 \pm 2,27 \mu \mathrm{S} / \mathrm{cm}$ - mantiveram-se dentro da faixa recomendada para a espécie (Popma \& Phelps, 1998).

Não foram observadas diferenças $(\mathrm{P}<0,05)$ nos parâmetros avaliados entre os diferentes tratamentos. As médias de peso final (PF), ganho de peso (GP), comprimento final $(\mathrm{CF})$, conversão alimentar aparente $(\mathrm{CA})$, fator de condição e taxa sobrevivência (SO) durante o período experimental encontram-se na Tabela 3 . 
Tabela 1 - Composição das rações experimentais (\%) Table 1 - Ingredient composition of the experimental diets (\%)

\begin{tabular}{|c|c|c|c|c|c|}
\hline \multirow[t]{2}{*}{$\begin{array}{l}\text { Ingrediente } \\
\text { Ingredient }\end{array}$} & \multicolumn{5}{|c|}{$\begin{array}{l}\text { Nível de energia digestível }(\mathrm{kcal} / \mathrm{kg}) \\
\text { Level of digestible energy }(\mathrm{kcal} / \mathrm{kg})\end{array}$} \\
\hline & 2900 & 3025 & 3150 & 3275 & 3400 \\
\hline Farelo de soja & 66,20 & 66,68 & 67,169 & 67,65 & 68,14 \\
\hline $\begin{array}{l}\text { Soybean meal } \\
\text { Milho }\end{array}$ & 28,84 & 25,99 & 23,14 & 20,29 & 17,44 \\
\hline Corn & & & & & \\
\hline $\begin{array}{l}\text { Óleo de soja } \\
\text { Soybean oil }\end{array}$ & 0,00 & 2,32 & 4,64 & 6,96 & 9,27 \\
\hline $\begin{array}{l}\text { Antioxidante (BHT) } \\
\text { Antioxidant (BHT) }\end{array}$ & 0,02 & 0,02 & 0,02 & 0,02 & 0,02 \\
\hline $\begin{array}{l}\text { Calcáreo calcítico } \\
\text { Limestone }\end{array}$ & 1,53 & 1,55 & 1,58 & 1,60 & 1,62 \\
\hline $\begin{array}{l}\text { Fosfato bicálcico } \\
\text { Dicalcium phosphate }\end{array}$ & 1,84 & 1,86 & 1,88 & 1,90 & 1,93 \\
\hline $\begin{array}{l}\text { Suplemento }(\text { Min. }+ \text { Vitam. })^{1} \\
\text { Supplement }(\text { Min }+ \text { Vit })^{I}\end{array}$ & ${ }^{1} 1,00$ & 1,00 & 1,00 & 1,00 & 1,00 \\
\hline $\begin{array}{l}\text { Sal comum } \\
\text { Salt }\end{array}$ & 0,50 & 0,50 & 0,50 & 0,50 & 0,50 \\
\hline
\end{tabular}

${ }^{1}$ Niveis de garantia por quilograma do produto (Rovimix peixes): Vit. A, 500.000 UI; Vit. D3, 200.000 UI; Vit. E, 5.000 mg; Vit. K3, 1.000 mg; Vit. B1, $1.500 \mathrm{mg}$; Vit. B2, $1.500 \mathrm{mg}$; Vit. B6, $1.500 \mathrm{mg}$; Vit. B12, $4.000 \mathrm{mg}$; Ác. fólico, $500 \mathrm{mg}$; Pantotenato Ca, $4.000 \mathrm{mg}$; Vit. C, $15.000 \mathrm{mg}$; Biotina, $50 \mathrm{mg}$; Inositol, 10.000; Nicotinamida, 7.000; Colina, $40.000 \mathrm{mg}$; Co, 10 mg; Cu, 500 mg; Fe, 5.000 mg; I, 50 mg; Mn, 1500 mg; Se, 10 mg; $\mathrm{Zn}, 5.000 \mathrm{mg}$

1 Guarantee levels for kilogram of the product (Rovimix fish): Vit. A, 500.000 UI; Vit. D3, 200.000 UI: Vit. E. $5.000 \mathrm{mg}$ : Vit. K3, $1.000 \mathrm{mg}$ : Vit. B1, $1.500 \mathrm{mg}:$ Vit. B2, $1.500 \mathrm{mg}$ Vit. B6, $1.500 \mathrm{mg}$; Vit. B12, $4.000 \mathrm{mg}$; Folic acid, $500 \mathrm{mg}$; Ca pantethonate, $4.000 \mathrm{mg}$; Vit. C, $15.000 \mathrm{mg}$; Biotin, $50 \mathrm{mg}$; Inosit, 10.000; Nicotinamid, 7.000; Hill, $40.000 \mathrm{mg} ; \mathrm{Co}, 10 \mathrm{mg}$; $\mathrm{Cu}, 500 \mathrm{mg} ; \mathrm{Fe}, 5.000 \mathrm{mg}: \mathrm{I}, 50 \mathrm{mg} ; \mathrm{Mn}, 1500 \mathrm{mg} ; \mathrm{If}, 10 \mathrm{mg} ; \mathrm{Zn}, 5.000 \mathrm{mg}$

Os parâmetros de desempenho $\mathrm{PF}, \mathrm{GP}, \mathrm{CF}, \mathrm{CA}, \mathrm{FC}$ e SO não apresentaram diferenças $(\mathrm{P}>0,05)$ em função dos diferentes níveis de energia das rações, demonstrando que as diferentes relações proteína:energia avaliadas não influenciaram o desempenho dos peixes. Vários autores relatam a importância da relação proteína:energia na formulação de rações para obtenção de bom desempenho dos animais (Chou \& Shiau, 1996; Lin et al., 1997; Meurer et al., 2002; Boscolo et al., 2002; Bell et al., 2003).

Os resultados obtidos neste experimento se assemelham aos observados por Chou \& Shiau (1996), Lovell (1989), El-Dahhar \& El-Shazly (1993) e Meurer et al. (2002), que afirmam que a energia proveniente dos lipídeos tem pouca influência no crescimento de tilápias. Conforme Boscolo et al. (2002, 2005) e Pezzato et al. (2002), a tilápia utiliza eficientemente a energia do milho, rico em amido e bem aproveitado pelas tilápias, inclusive na fase inicial (Chou \& Shiau, 1996 e Lin et al., 1997).

Neste experimento a relação amido:lipídeo na dieta variou de 14,1 a 1,87 com o aumento dos níveis de energia, não se observando diferenças ( $\mathrm{P}>0,05)$ no desempenho dos alevinos. Resultados semelhantes foram encontrados por Chou \& Shiau (1996), que observaram variação na relação amido:lipídeo na dieta de 1,13 a 8,4 e não constataram diferenças $(\mathrm{P}>0,05)$ no desempenho dos alevinos nos diferentes
Tabela 2 - Composição química das rações experimentais Table 2 - Chemical composition of the experimental diets

\begin{tabular}{|c|c|c|c|c|c|}
\hline \multirow[t]{2}{*}{$\begin{array}{l}\text { Nutriente }(\%) \\
\text { Nutrient }\end{array}$} & \multicolumn{5}{|c|}{$\begin{array}{c}\text { Nível de energia digestível }(\mathrm{kcal} / \mathrm{kg}) \\
\text { Level of digestible energy }(\mathrm{kcal} / \mathrm{kg})\end{array}$} \\
\hline & 2.900 & 3.025 & 3.150 & 3.275 & 3.400 \\
\hline $\begin{array}{l}\text { Energia digestível } \\
(\mathrm{kcal} / \mathrm{kg})^{1} \\
\text { Digestible energy }{ }^{1}\end{array}$ & 2.900 & 3.025 & 3.150 & 3.275 & 3.400 \\
\hline $\begin{array}{l}\text { Proteina bruta } \\
\text { Crude protein }\end{array}$ & 33,54 & 33,53 & 33,56 & 33,57 & 33,58 \\
\hline $\begin{array}{l}\text { Proteina digestível }{ }^{2} \\
\text { Digestible protein }{ }^{2}\end{array}$ & 30,00 & 30,00 & 30,00 & 30,00 & 30,00 \\
\hline $\begin{array}{l}\text { Gordura } \\
\text { Fat }\end{array}$ & 1,91 & 4,11 & 6,32 & 8,52 & 10,73 \\
\hline $\begin{array}{l}\text { Fibra bruta } \\
\text { Crude fiber }\end{array}$ & 4,47 & 4,45 & 4,42 & 4,40 & 4,37 \\
\hline $\begin{array}{l}\text { Fósforo total } \\
\text { Total phosphorus }\end{array}$ & 0,80 & 0,80 & 0,80 & 0,80 & 0,80 \\
\hline $\begin{array}{l}\text { Cálcio } \\
\text { Calcium }\end{array}$ & 1,15 & 1,16 & 1,17 & 1,19 & 1,20 \\
\hline $\begin{array}{l}\text { Amido } \\
\text { Starch }\end{array}$ & 26,90 & 25,19 & 23,48 & 21,77 & 20,06 \\
\hline $\begin{array}{l}\text { Metionina }+ \text { cistina } \\
\text { Met }+ \text { Cys }\end{array}$ & 1,22 & 1,22 & 1,21 & 1,21 & 1,21 \\
\hline $\begin{array}{l}\text { Acido linolênico } \\
\text { Lynoleic acid }\end{array}$ & 1,03 & 2,23 & 3,43 & 4,63 & 5,83 \\
\hline $\begin{array}{l}\text { Lisina } \\
\text { Lysine }\end{array}$ & 1,91 & 1,92 & 1,93 & 1,93 & 1,94 \\
\hline $\begin{array}{l}\text { Metionina } \\
\text { Methionine }\end{array}$ & 0,55 & 0,55 & 0,55 & 0,55 & 0,55 \\
\hline $\begin{array}{l}\text { Treonina } \\
\text { Threonine }\end{array}$ & 1,27 & 1,27 & 1,27 & 1,27 & 1,27 \\
\hline $\begin{array}{l}\text { Triptofano } \\
\text { Thryptophan }\end{array}$ & 0,45 & 0,45 & 0,45 & 0,45 & 0,45 \\
\hline $\begin{array}{l}\text { Valina } \\
\text { Valine }\end{array}$ & 1,53 & 1,52 & 1,52 & 1,52 & 1,52 \\
\hline $\begin{array}{l}\text { Leucina } \\
\text { Isoleucina }\end{array}$ & $\begin{array}{l}2,64 \\
1,48\end{array}$ & $\begin{array}{l}2,62 \\
1,48\end{array}$ & $\begin{array}{l}2,62 \\
1,48\end{array}$ & $\begin{array}{l}2,60 \\
1,49\end{array}$ & $\begin{array}{l}2,59 \\
1,49\end{array}$ \\
\hline $\begin{array}{l}\text { Isoleucyne } \\
\text { Histidina } \\
\text { Hystidine }\end{array}$ & 0,85 & 0,85 & 0,84 & 0,84 & 0,84 \\
\hline $\begin{array}{l}\text { Fenilalanina } \\
\text { Phenilalanine }\end{array}$ & 1,64 & 1,64 & 1,64 & 1,64 & 1,64 \\
\hline $\begin{array}{l}\text { Arginina } \\
\text { Arginine }\end{array}$ & 2,32 & 2,32 & 2,33 & 2,33 & 2,34 \\
\hline
\end{tabular}

${ }^{1}$ Baseados nos valores de energia digestível propostos por Boscolo et al (2002): farelo de soja $=3.057$; milho $=3.037$ e óleo de soja $=8.485 \mathrm{kcal} / \mathrm{kg}$

1 Based on the values of digestible energy by Boscolo et al. (2002). Soybean meal $=3,057$ corn $=3,037$ and soy oil $=8,485 \mathrm{kcal} / \mathrm{kg}$.

2 Baseados nos valores de proteína digestível propostos por Boscolo et al. (2002). Farelo de soja $=42,19$; milho $=7,18 \%$.

${ }^{2}$ Based on the values of digestible protein by Boscolo et al. (2002). Soybean meal $=42.19$; corn $=7.18$.

tratamentos com a inclusão de lipídeos, demonstrando que a relação amido:lipídeo superior a 1 pode ser benéfica ao desempenho de alevinos de tilápia-do-nilo (O. niloticus) $\mathrm{e}$ que altos níveis de amido facilitam a flutuação das rações submetidas ao processamento por extrusão (Kearns 1998).

Boscolo et al. (2005), no entanto, alteraram a relação amido-lipídeo de 1,09-0,11, por meio do aumento dos níveis de energia, e notaram que a relação amido:lipídeo igual ou inferior a 0,41 prejudicou o desenvolvimento das larvas, demonstrando que, mesmo na fase inicial, a relação 
Tabela 3 - Desempenho de alevinos de tilápia-do-nilo alimentados com diferentes níveis de energia digestível na dieta Table 3 - Performance of Nile tilapia fingerlings fed diets containing different levels of digestible energy

\begin{tabular}{|c|c|c|c|c|c|c|}
\hline \multirow[b]{2}{*}{$\begin{array}{l}\text { Parâmetro* } \\
\text { Parameter }\end{array}$} & \multicolumn{5}{|c|}{$\begin{array}{l}\text { Nível de energia digestível }(\mathrm{kcal} / \mathrm{kg}) \\
\text { Level of digestible energy }(\mathrm{kcal} / \mathrm{kg})\end{array}$} & \multirow[b]{2}{*}{$\mathrm{CV} \%$} \\
\hline & 2.900 & 3.025 & 3.150 & 3.275 & 3.400 & \\
\hline Peso inicial médio $(\mathrm{g})$ (Average initial weight) & 0,62 & 0,62 & 0,62 & 0,62 & 0,62 & 1,77 \\
\hline Peso final médio (g) (Average final weight) & 1,69 & 1,76 & 2,00 & 1,82 & 1,86 & 13,49 \\
\hline Ganho de peso médio ( $\mathrm{g}$ ) (Average weight of gain) & 1,07 & 1,15 & 1,39 & 1,20 & 1,25 & 20,20 \\
\hline Fator de condição (Condiction factor) & 3,81 & 3,85 & 3,82 & 3,88 & 4,17 & 9,06 \\
\hline Sobrevivência (\%) (Survival) & 80,00 & 88,00 & 80,00 & 92,00 & 84,00 & 17,65 \\
\hline
\end{tabular}

${ }^{*} \mathrm{P}>0,05$

amido:lipídeo como fonte de energia deve ser considerada para a obtenção de bom desempenho de $O$. niloticus.

Hayashi et al. (1999b) testaram diferentes níveis de lipídeos para alevinos de carpa-capim (Ctenopharyngodon idella) e observaram redução linear no desempenho dos animais à medida que aumentaram os níveis de lipídeo na ração. Meer et al. (1997) não registraram diferenças no desempenho de tambaqui (Colossoma macropomum) alimentados com diferentes níveis de lipídeos, o que demonstra variações nos resultados com a utilização de lipídeo e amido como fontes de energia pelas diferentes espécies.

Não foram observadas diferenças $(\mathrm{P}>0,05)$ na $\mathrm{CA}$ entre os diferentes tratamentos, o que comprova que a tilápia aproveita eficientemente a energia disponível na dieta. Resultados contrários ao deste experimento foram reportados por Meurer et al. (2002), que observaram redução linear na CA de alevinos de tilápia-do-nilo com a inclusão de lipídeos na dieta, porém esses autores mantiveram as rações isoenergéticas. Martino et al. (2002), por sua vez, avaliaram rações com diferentes níveis de óleo de soja e, portanto, com aumento nos níveis energéticos para alevinos de pintado (Pseudoplatistoma corruscans) e observaram melhores valores de CA para os peixes alimentados com rações contendo altos teores de óleo de soja e energia.

Resultados de CA semelhantes aos obtidos neste experimento foram encontrados por Chou \& Shiau (1996), que também não observaram diferenças $(\mathrm{P}>0,05)$ para esse parâmetro. Portanto, para uma boa $\mathrm{CA}$ de alevinos de tilápiado-nilo, é necessário considerar a relação amido/lipídeo na formulação de rações.

A principal forma de armazenagem de energia corporal é como gordura, por suas características de hidrofobicidade e de fácil acondicionamento nas moléculas de triacilgliceróis no interior dos adipócitos (Lehninger et al., 1995). A energia não é um nutriente, mas resulta da oxidação dos nutrientes durante o metabolismo e pode ser liberada na forma de calor ou armazenada para uso posterior nos processos metabó- licos do organismo animal (NRC, 1993). É fundamental a participação de fontes energéticas na formulação de rações, mas a relação energia:proteína e amido:lipídeo na dieta deve ser balanceada para que os peixes apresentem bom desempenho.

\section{Conclusões}

Recomenda-se a utilização de 2.900 a $3.400 \mathrm{kcal}$ de ED $/ \mathrm{kg}$ de ração para alevinos de tilápia-do-nilo (O. niloticus). Para obtenção de bom desempenho dos peixes, a relação amido:lipídeo como fonte de energia deve ser de 14,1 a 1,87 na formulação de rações.

\section{Literatura Citada}

BARROS, M.M.; PEZZATO, L.E.; KLEEMANN, G.K. et al. Níveis de vitamina $\mathrm{C}$ e ferro para tilápia do Nilo (Oreochromis niloticus). Revista Brasileira de Zootecnia, v.31, n.6, p.2149-2156, 2002.

BELL, J.G.; MEcGHEE, F.; CAMPBELL, P.J. Rpeseed oil an alternative to marine fish oil in diets of post-smolt Atlantic salmon (Salmo salar): Changes in fish fatty acid composition and effectiveness of subsequent fish oil "wash out". Aquaculture, v.218, p.515-528, 2003.

BELLAL, J.E.H.; ASSEM, H. Substution of soybean meal and oil for fish meal in pratical diets fed to channel catfish, Ictalurus punctatus (Rafinesque): effects on body composition. Aquaculture Research, v.26, p.141-145, 1995.

BENDIKESEN, E.A.; ARNESEN, A.M.; JOBING, M. Effects of dietary fatty acid profile and fat content on smolting and seawater performance in Atlantic salmom (Salmo salar L.). Aquaculture, v.225, p.149-163, 2003.

BOSCOLO, W.R.; HAYASHI, C.; SOARES, C.M. et al. Desempenho e características de carcaça de machos revertidos de tilápias do Nilo (Oreochromis niloticus), linhagens tailandesa e comum, nas fases iniciais e de crescimento. Revista Brasileira de Zootecnia, v.30, n.5, p.1391-1396, 2001

BOSCOLO, W.R.; HAYASHI, C.; MEURER, F. Digestibilidade aparente da energia e nutrientes de alimentos convencionais e alternativos para a tilápia do Nilo (Oreochromis niloticus, L.). Revista Brasileira de Zootecnia, v.13, n.2, p.539-545, 2002 BOSCOLO, W.R.; HAYASHI, C.; MEURER, F. et al. Digestibilidade aparente da energia e proteína das farinhas de resíduo da filetagem da tilápia do Nilo (Oreochromis niloticus) e da corvina 
(Plagioscion squamosissimus) e farinha integral do camarão canela (Macrobrachium amazonicum) para a tilápia do Nilo. Revista Brasileira de Zootecnia, v.33, n.1, p. 8-13, 2004.

BOSCOLO, W.R.; SIGNOR, A.; FEIDEN, A. et al. Energia digestível para larvas de tilapia-do-nilo Oreochromis niloticus na fase de reversão sexual. Revista Brasileira de Zootecnia, v.34, n.6, p.1813-1818, 2005

CHOU, B.S.; SHIAU, S.Y. Optinal dietary lipid level for growth of juvenile hibrid tilápia, Oreochromis niloticus x Oreochromis niloticus aureus. Aquaculture, v.143, n.2, p.185-195, 1996.

EL-DAHHAR, A.A.; EL-SHAZLY, K. Effsct of essential amino acids ( methionine and lysine) and treated oil in fish diet on growth performace and feed utilization of Nile tilapia, Tilapia nilotica (L.) Aquaculture and Fishieries Managenent, v.24, n.6, p.731-739, 1993.

HANLEY, F. The digestibility of foodstuffs and the effects of feeding selectivity determinations in tilapias (Oreochromis niloticus L.). Aquaculture, v.66, n.2, p.163-179, 1987.

HAYASHI, C.; BOSCOLO, W.R.; SOARES, C.M. et al Exigência de proteína digestivel para larvas de tilápia do Nilo (Oreochromis niloticus), durante a reversão sexual. Revista Brasileira de Zootecnia, v.31, n.2, p.823-828, 2002.

HAYASHI, C.; MEURER, F.; SOARES, C.M. et al. Utilização de diferentes níveis de gordura em dietas para alevinos de carpa capim (Ctenopharingodon idella, V) In: CONGRESSO BRASILEIRO DE ENGENHARIA DE PESCA, 11., $1999 \mathrm{~b}$. 1999b. Olinda. Anais... Olinda: Congresso Brasileiro de Engenharia de Pesca: 1999b. p.201-210

HAYASHI,C.; BOSCOLO,W.R.; SOARES,C.M. et al. Uso de diferentes graus de moagem dos ingredientes em dietas para a tilapia do Nilo (Oreochromis niloticus L.) na fase de crescimento. Acta scientiarum, v.21, n.3, p.733-737, 1999a.

HILDSORF, A.W.S. Genética e cultivo de tilápias vermelhas, uma revisão. Boletim Instituto de Pesca, v.22, n.1, p.73-78, 1995.

JAUNCEY, K. Nutricional requirements. In: BEVERIDGE, M.C.M.; McANDREW, B.J. (Eds.) Tilapias: biology and exploitation. Great Britain: Kluwer Academic Publishers, 2000. p.327-375.

KEARNS, J.P. Ingredient consideration, preparation of feeds, selection of extrusion equipament and automaticcontrols for extrusion of pet and aquatic feedes. In: ADVANCES IN EXTRUSION TECHNOlOGY, 1., 1998, São Paulo. Anais... São Paulo: Faculdade de Engenharia de Alimentos, Universidade Estadual de Campinas, 1998. p.97-105.

LEE, S.M.; LEE, J.H.; KIM, K.D. Effect of dietary essential fatty acids on growth, body composition and blood chemistry of juvenile starry flounder (Platichthys stellatus). Aquaculture. Amsterdam, v.225, p.269-281, 2003

LEHNIINGER, A.L.; NELSON, D.L.; COX, M.M. Princípios de Bioquímica. São Paulo: Sarvier, 1995. 839p.

LIN, J.H.; CUI, Y.; HUNG, S.S.O. et al. Effect of feeding strategy and carbohydrate source on carbohydrate utilization by white sturgeon (Acipenser transmontanus) and hybrid tilapia (Oreochromis niloticus x O. aureus). Aquaculture, v.148, p.201-211, 1997.

LOWELL, T. Nutiriton and feeding of fish. New York:Van Nostrand Reinhold, 1989. p.11-18.
LUTZ, C.G. Production economics and potential competitive dynamics of commercial tilapia culture in the Americas. In COSTA-PIERCE, B.A.; RAKOY, J.E. (Eds.) Tilapia aquaculture in the Americas. Baton Rouge: World Aquaculture Society, 1995. v.2, p.119-132.

MARTINO, C.R.; CYRINO, J.E.P.; PORTZ, L. et al. Effect of dietary lipid level on nutricional performance of surubim (Pseudoplatistoma corruscans). Aquaculture, v.209, p.209218, 2002

MCGOOGAN, B.B.; REIGH, R.C. Apparent digestibility of selected ingredients in red drum (Sciaenops ocellatus) diets. Aquaculture, v.14, p.233-244, 1996.

MEER, M.B.; ZAMORA, J.E.; VERDEGEM, M.C.J. Effect of dietary lipid level on protein utilization one the size and proximate composition of fody compartments of Colossoma macropomum (Cuvier). Aquaculture Research, v.28, n.6, p.405-417, 1997.

MENTE, M.M.; SIGNOR, A.; FRECCIA, A. et al. Investigação de salmonella em diferentes sistemas de cultivo de tilápia do Nilo (Oreochromis niloticus) In: CONGRESSO BRASILEIRO DE ENGENHARIA DE PESCA, 13., 2003. Anais... Congresso Brasileiro de Engenharia de Pesca, 2003. p.1104-1109.

MEURER, F.; HAYASHI, C.; BOSCOLO, W.R. et al. Lipídeos na alimentação de alevinos revertidos de tilápia do Nilo (Oreochromis niloticus L.). Revista Brasileira de Zootecnia, Viçosa, v.31, n.2, p.566-573, 2002.

MUKHOPADHYAY, N.; RAY, A. K. The apparent total and nutrient digestibility of sai seed (Shorea robusta) meal in rohu, Labeo rohita (Hamilton), fingerlings. Aquaculture Research, v.28, p.683-689, 1997.

NATIONAL RESEACH COUNCIL - NRC. Nutrient requirements of fish. Washington, D.C.: National Academic Press, 1993. $114 \mathrm{p}$.

PEZZATO, L.E.; MIRANDA, E.C.; BARROS, M.M. et al. Digestibilidade aparente de ingredientes pela tilápia do Nilo (Oreochromis niloticus). Revista Brasileira de Zootecnia v.31, n.4, p.1595-1604, 2002.

POPMA, T.J.; PHELPS, R.P. Status report to commercial Tilapia producers on monosex fingerling production techniques. In: AQUICULTURA BRASIL, 1., 1998, Recife. Anais... Recife: Simpósio Brasileiro de Aquicultura, 1998. p.127-145.

SANCHES, L.E.F. Substituição do óleo de soja por óleo de tilápia e óleo de visceras de aves em rações para alevinos de tilápia do Nilo (Oreochromis niloticus). Maringá: Universidade Estadual de Maringa, 2004. 79p. Tese (Doutorado em Zootecnia) - Universidade Estadual de Maringá, 2004.

STEFFENS, W. Princípios fundamentales de la alimentación de los peces. Madri: Acribia, 1987. 275p.

UNIVERSIDADE FEDERAL DE VIÇOSA - UFV. SAEG - Sistema para Análises Estatísticas e Genéticas. Versão 7.1 Viçosa, MG: 1997. 150p. (Manual do usuário). 